\title{
Eventos adversos e segurança do paciente no atendimento de urgência e emergência pré-hospitalar
}

\author{
Adverse events and patient safety in emergency care and pre-hospital emergency \\ Eventos adversos y seguridad del paciente en atención de emergencia y emergencia \\ prehospitalaria
}

\begin{abstract}
Wellington Manoel da Silva ${ }^{1 *}$, Maria Eduarda da Silva ${ }^{2}$, Willaine Balbino de Santana Silva ${ }^{3}$, Simone Souza de Freitas ${ }^{2}$, Danielly Alves Mendes Barbosa ${ }^{2}$, Williane Souza da Silva ${ }^{2}$, Jessika Luana da Silva Albuquerque ${ }^{3}$, Midian Beatriz de Oliveira ${ }^{2}$, Mariany Stefany de Freitas Nascimento ${ }^{3}$, Georgia Cybelle dos Santos Silva ${ }^{3}$.
\end{abstract}

\section{RESUMO}

Objetivo: Analisar como se encontra a publicação científica acerca das causas relacionadas a ocorrência de eventos adversos nos serviços de urgência e emergência pré-hospitalar. Métodos: Trata-se de uma revisão integrativa da literatura, com buscas realizadas nas bases de dados Biblioteca Virtual de Saúde. Os descritores utilizados foram "Segurança do paciente", "Enfermagem em Emergência" e "Eventos Adversos", usando o conectivo boleano "and" para realizar o cruzamento das palavras-chave, utilizou-se ainda, como critérios de inclusão, artigos publicados no período de 2012 a 2018 e trabalhos completos. Resultados: Desde o final da década de 90, tem-se estudado um fenômeno denominado de eventos adversos (EAs), o qual é responsável por índices de saúde alarmantes nos países em que foi investigado. É estimado que em torno de 100 mil indivíduos morram a cada ano, em decorrência de EAs nos Estados Unidos da América (EUA). Como resultado, a taxa de mortalidade, nos EUA, devido a eventos adversos, é maior que outras, tais como AIDS, câncer de mama ou atropelamentos. Considerações finais: A capacitação dos profissionais, através de treinamentos em serviços de educação em saúde, além da padronização das rotinas protocoladas pode se caracterizar como um norteador para promover o alinhamento entre rotinas e prática profissional.

Palavras-chave: Segurança do paciente, Enfermagem em emergência, Eventos adversos.

\section{ABSTRACT}

Objective: To analyze how the scientific publication is found on initiatives that promote greater safety for patients seen in urgent and pre-hospital emergency services. Methods: This is an integrative review of the literature, with searches performed in the Virtual Health Library. The descriptors used were "patient safety", "emergency nursing" and "Adverse Events", using the Boolean connective "and" to perform the crossing of keywords, articles published in the period from 2012 to 2018 and complete works. Results: Since the late 1990s, a phenomenon called adverse events (AEs) has been studied, which is responsible for alarming health rates in the countries in which it was investigated. It is estimated that around 100,000 individuals die each year as a result of AEs in the United States of America (USA). As a result, the mortality rate in the USA due to adverse events is higher than others, such as AIDS, breast cancer or being run over. Final considerations: The training of professionals, through training in health education services, in addition to the standardization of protocoled routines, can be characterized as a guide to promote the alignment between routines and professional practice.

Keywords: Patient safety, Emergency nursing, Adverse events.

\footnotetext{
${ }^{1}$ Instituto de Medicina Integral Professor Fernando Figueira (IMIP), Recife - PE.

*E-mail: wellington-manoel@outlook.com

2Universidade Federal de Pernambuco (UFPE), Vitória de Santo Antão - PE.

3Universidade de Pernambuco (UPE), Recife - PE.

${ }^{4}$ Centro Universitário da Vitória de Santo Antão (UNIVISA), Vitória de Santo Antão - PE.
}

PUBLICADO EM: 9/2020 


\section{RESUMEN}

Objetivo: Analizar cómo se encuentra la publicación científica en iniciativas que promueven una mayor seguridad para los pacientes tratados en servicios de urgencias urgentes y prehospitalarios. Métodos: Esta es una revisión integradora de la literatura, con búsquedas realizadas en las bases de datos Biblioteca Virtual de Salud. Los descriptores utilizados fueron "seguridad del paciente", "enfermería de emergencia" y "Eventos adversos", utilizando el conectivo booleano "y" para realizar el cruce de palabras clave, artículos publicados en el período de 2012 a 2018 y obras completas. Resultados: Desde finales de la década de 1990, se ha estudiado un fenómeno llamado eventos adversos (EA), que es responsable de las alarmantes tasas de salud en los países en los que se investigó. Se estima que alrededor de 100 mil personas mueren cada año como resultado de EA en los Estados Unidos de América (EUA). Como resultado, la tasa de mortalidad en los EUA. Debido a eventos adversos es más alta que otras, como el SIDA, el cáncer de mama o el atropello. Consideraciones finales: La capacitación de profesionales, a través de la capacitación en servicios de educación para la salud, además de la estandarización de las rutinas protocoladas, puede caracterizarse como una guía para promover la alineación entre las rutinas y la práctica profesional.

Palabras clave: Seguridad del paciente, Enfermería de emergencia, Eventos adversos.

\section{INTRODUÇÃO}

No Brasil, o Sistema de Saúde, de acesso universal e igualitário, é regido por meio de políticas que atribuem ao Estado o dever de garantir a prevenção, recuperação e promoção à saúde. Desta forma, o Sistema único de Saúde (SUS) é organizado, a fim de garantir o acesso aos serviços de saúde, de forma integral e igualitária, hierarquizada e regionalizada em níveis crescentes de complexidade no atendimento (SILVA HC, 2016). A assistência à saúde no contexto do SUS é estabelecida por meio da organização das ações e dos procedimentos em rede, compreendendo três níveis de complexidade: atenção primária, média complexidade e alta complexidade (OLIVEIRA SN, 2015).

Esse sistema de redes de atenção em saúde é sustentado por critérios, fluxos e pactuações de funcionamento, que asseguram o princípio da integralidade aos usuários e por meio de um desenho lógico evitam encaminhamentos desnecessários, tornado a atenção em saúde mais resolutiva (OLIVEIRA SN, 2015). Neste contexto, as políticas que estabelecem a rede de urgência e emergência, demandam uma atenção diferenciada, devido à demanda por atendimentos em função do aumento do número de acidentes, da violência e das fragilidades da estruturação da rede permanecerem problemas constantes, gerando superlotação em unidades hospitalares e sobrecarga de trabalho para os profissionais.

Tais especificidades apontam para altos índices de rotatividade de recursos humanos e insatisfação com o ambiente de trabalho, podendo gerar impactos negativos para a qualidade da assistência prestada (CUNHA VP, 2020).

O atendimento pré-hospitalar (APH) móvel é um serviço que integra a rede de atenção às urgências, embora seja considerado relativamente recente no Brasil, ressalta-se sua relevância e contribuição no atendimento às urgências e emergências (CUNHA VP, 2020).

O Atendimento pré-hospitalar (APH), realizado pelo Serviço Móvel de Urgência e Emergência (SAMU), é o primeiro atendimento prestado por profissionais da saúde, em situações ocorridas fora do hospital, em casos de traumas resultantes de acidentes automobilísticos, distúrbios em pacientes crônicos, mal súbito de qualquer etiologia e/ou distúrbios psiquiátricos de maneira que, visa à estabilização clínica do paciente no local do ocorrido e em seguida sua segura remoção para uma unidade hospitalar adequada ao quadro clínico apresentado pela vítima (RIBEIRO AC, 2016; SANTOS NCM, 2010; CRESPILHO DF, et al., 2015).

O APH objetiva a execução de intervenções rápidas, seguras e com o intuito de evitar possíveis sequelas transitórias ou permanentes, além de possibilitar uma maior sobrevida ao paciente, por diminuir o período sem atendimento às vítimas de agravos à saúde de qualquer natureza, sendo assim, a preocupação acerca da segurança do paciente norteia todos os níveis da assistência (CUNHA VP, et al., 2019). 
Esta preocupação ganhou volume internacionalmente após a publicação do Relatório To err is Human: Building a safer Health System, no qual o Committee on Quality of Health Care in America, revelou as situações de risco relacionado à assistência em que os pacientes foram expostos no decorrer do atendimento de urgência e emergência extra-hospitalar no sistema de saúde americano (CRESPILHO DF, et al., 2015).

No ano de 2013, o Ministério da Saúde, (MS) por intermédio da Agência Nacional de Vigilância Sanitária (ANVISA), visando estimular práticas seguras durante a assistência em saúde ao paciente publicou um caderno de ações de segurança do paciente, pontuando áreas em que haja possibilidade recorrente de erros durante o processo do cuidado (BRASIL, 2013). Para Castro et., al (2018) o conceito de segurança do paciente compreende medidas preventivas, afim de evitar possíveis agravos ocorridos durante o processo assistencial aos pacientes e demais sujeitos envolvidos neste processo, que devido a complexa rede de eventos que envolvem o cuidado, tornam-se susceptíveis a ocorrências de erros (CASTRO GLT, et al., 2018).

Erros ou incidentes configuram-se como eventos ou ocorrência cujo resultado foi algum tipo de dano evitável ao paciente, proveniente de ações intencionais ou não (DUARTE et al., 2015). A World Health Organization (WHO) publicou, no ano de 2012, um material que afirma que quando estes erros não atingem direta ou indiretamente o paciente, podem ser denominados de quase erro, quando o atingem, porém não lhe causando nenhum dano identificável, são chamados de incidente sem danos, e quando resultam em dano visível ou identificável, são denominados de eventos adversos (EA) (WHO, 2012).

O estudo tem como objetivo analisar como se encontra a publicação científica a fim de identificar as possíveis causas da ocorrência de acidentes entendidos aqui como Eventos Adversos durante o processo do cuidado de urgência e emergência extra-hospitalar no intuito de que possa por meios dos resultados apresentados colaborar para a promoção de maior segurança do paciente atendido nos serviços de urgência e emergência pré-hospitalar.

\section{MÉTODOS}

Foi realizada uma revisão integrativa da literatura, com o objetivo de identificar, analisar e sintetizar resultados de estudos a fim de apresentar como se encontra a publicação científica acerca de iniciativas que promovam a maior segurança do paciente atendido nos serviços de urgência e emergência. $A$ revisão integrativa reúne e sintetiza resultados de pesquisas de maneira sistemática e organizada, sobre um delimitado tema contribuindo dessa forma com o aprofundamento do conhecimento acerca do tema investigado, sendo assim, a revisão integrativa não é mera repetição do que já existe escrito sobre determinado assunto, mas proporciona o exame de um tema sob nova perspectiva ou abordagem, possibilitando conclusões inovadoras, motivo pelo qual se escolheu esse método (LAKATOS EM e MARCONI MA, 2003).

Foram utilizadas as seguintes etapas recomendadas: seleção de questão norteadora; definição das características das pesquisas da amostra; seleção das pesquisas que compuseram a amostra da revisão; análise dos achados dos artigos incluídos na revisão; interpretação dos resultados e exame crítico dos achados (GALVÃO TF e PEREIRA MG, 2014). Para orientar este estudo, estabeleceu-se a questão norteadora: $O$ que há na literatura acerca das causas motivadoras de eventos adversos, durante o processo de cuidado no atendimento pré-hospitalar de emergência?

O levantamento de artigos foi realizado de agosto a outubro de 2019, por meio do acesso on-line no sítio da Biblioteca Virtual de Saúde (BVS), onde a averiguação dos artigos é feita de forma ampla, utilizando todas as bases de dados ali contidas, a saber: Literatura Latino-Americana e do Caribe em Ciências da Saúde (LILACS), biblioteca digital Scientific Electronic Library Online (SciELO), Índice Bibliográfico Espanhol em Ciências da Saúde (IBECS) e Medical Literature Analysis and Retrieval System Online (Medline), entre outras, utilizando-se os descritores dos Descritores em Ciências da Saúde (DeCS) da Biblioteca Virtual em Saúde e do Medical Subject Headings (MeSH) da National Library nos idiomas português, inglês ou espanhol: segurança do paciente, enfermagem em emergência e Eventos Adversos, usando o conectivo boleano "and" para realizar o cruzamento das palavras-chave, utilizou-se ainda, como critérios de inclusão, artigos publicados no período de 2012 a 2018 e trabalhos completos. 
Foram excluídos, editoriais, artigos que não respondessem à pergunta condutora, incompletos, cartas ao editor, dissertações, teses e artigos repetidos. Para análise quantitativa, fez-se uso de fichamento dos artigos contendo título, autores, ano de publicação, periódico, base de dados e extrato Qualis do periódico.

\section{RESULTADOS E DISCUSSÃO}

Do total de 627 trabalhos encontrados, foram excluídos 622, editoriais, artigos que não respondessem à pergunta condutora (594), incompletos (4), cartas ao editor, dissertações, teses e artigos repetidos (24), desta forma, foram selecionados quatro artigos em português, conforme mostra o fluxograma da Figura 1.

Figura 1 - Fluxograma de Seleção dos artigos para a análise.

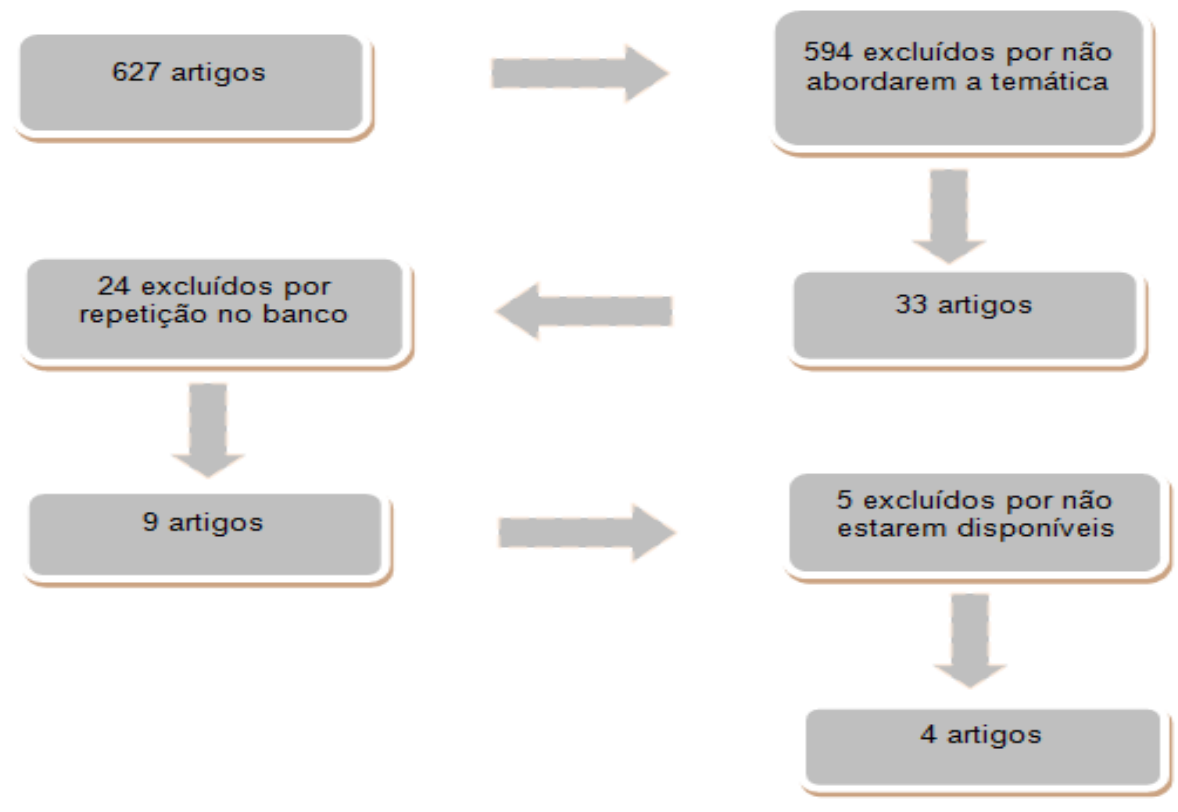

Fonte: Silva WM, et al., 2020.

As informações selecionadas para a caracterização dos estudos foram: autor, ano de publicação, periódico, título do estudo e base de dados e estão representadas no Quadro 1.

Quadro 1- Distribuição dos estudos de acordo com o título, autores, periódicos, ano de publicação, base de dados e extrato Qualis Capes.

\begin{tabular}{|c|c|c|c|c|c|c|}
\hline $\mathbf{N}$ & $\begin{array}{c}\text { Título } \\
\end{array}$ & Autores & Periódico & Ano & Bases & Qualis \\
\hline 1 & $\begin{array}{l}\text { Percepção da } \\
\text { enfermagem sobre os } \\
\text { fatores de risco que } \\
\text { envolvem a segurança do } \\
\text { paciente pediátrico. }\end{array}$ & SOUZA FT, et al. & $\begin{array}{l}\text { Revista de } \\
\text { Enfermagem da } \\
\text { UFSM. }\end{array}$ & 2014 & Bireme & B2 \\
\hline 2 & $\begin{array}{l}\text { Segurança do paciente } \\
\text { na assistência pré- } \\
\text { hospitalar de emergência: } \\
\text { uma revisão integrativa. }\end{array}$ & CRESPILHO DF, et al. & $\begin{array}{c}\text { Revista Uningá } \\
\text { Review }\end{array}$ & 2015 & $\begin{array}{l}\text { Google } \\
\text { Scholar }\end{array}$ & B4 \\
\hline 3 & $\begin{array}{l}\text { Cultura de segurança do } \\
\text { paciente na perspectiva } \\
\text { da equipe de enfermagem } \\
\text { de emergências } \\
\text { pediátricas. }\end{array}$ & MACEDO TR, et al. & $\begin{array}{c}\text { Revista Escola } \\
\text { de Enfermagem } \\
\text { da USP. }\end{array}$ & 2016 & Lilacs & A2 \\
\hline 4 & $\begin{array}{l}\text { Instrumento para } \\
\text { avaliação da qualidade da } \\
\text { assistência pré-hospitalar } \\
\text { móvel de urgência: } \\
\text { validação de conteúdo. }\end{array}$ & $\begin{array}{l}\text { DANTAS RAN e } \\
\text { TORRES GV; } \\
\text { SALVETTI MG; } \\
\text { DANTAS DV e } \\
\text { MENDONÇA AEO. }\end{array}$ & $\begin{array}{c}\text { Revista Escola } \\
\text { de Enfermagem } \\
\text { da USP. }\end{array}$ & 2015 & SciELo & A2 \\
\hline
\end{tabular}

Fonte: Silva WM, et al., 2020 
Três dos quatro trabalhos selecionados são de autoria exclusiva de enfermeiros e o último foi escrito por equipe Multidisciplinar formada por enfermeiros e médicos, o que revela um crescente interesse da enfermagem nessa temática, um estudo que revisou artigos científicos publicados no período de 2000 a 2014 nas bases de dados da Biblioteca Virtual em Saúde, buscando encontrar como se encontrava a publicação científica acerca de medidas que visassem produzir segurança no paciente durante 0 atendimento de urgência, Identificou 180 trabalhos, destes, apenas quatro artigos foram selecionados para análise e destes apenas um, exclusivamente foi de autoria de enfermeiros (CRESPILHO DF, et al., 2015).

No que concerne ao período de divulgação dos artigos, os quatro trabalhos foram publicados entre os anos de 2012 e 2016. Dois trabalhos são originários do mesmo periódico internacional e dois trabalhos foram localizados em periódicos nacionais. Dois trabalhos estão disponíveis nos idiomas inglês e português e outros dois apenas no idioma português. Dois artigos foram publicados em periódicos com estrato Qualis $A$ e dois em periódicos com extrato Qualis B, o que classifica os dois primeiros trabalhos com recomendação forte, apontando para grande evidência científica, em contrapartida, os últimos classificam-se como média/baixa evidenciação científica e são apenas recomendados.

Os estudos analisados tiveram como tema central a segurança do paciente, desta forma todos, à sua visão, trouxeram contribuições relativas às medidas de prevenção de eventos adversos no contexto da assistência de urgência e emergência no ambiente pré-hospitalar, portanto, permitindo identificar quais as causas dessas ocorrências.

Desde o final da década de 90, pesquisadores de vários países, a saber, Estados Unidos da América (EUA), Canadá, Dinamarca, França, Austrália, Nova Zelândia, Inglaterra, Espanha, Brasil, Suécia e Holanda tem estudado de forma sistemática um fenômeno intrinsecamente relacionado a problemas de qualidade de assistência em serviços de saúde. Esse fenômeno denominado de eventos adversos (EAs) é responsável por índices de saúde alarmantes nos países em que foi investigado (PORTO S, et al., 2010; MENDES W, et al., 2005).

É estimado que em torno de 100 mil indivíduos morram a cada ano, em decorrência de EAs nos EUA. Como resultado, a taxa de mortalidade, nos EUA, devido a eventos adversos, é maior que outras, tais como AIDS, câncer de mama ou atropelamentos (MENDES W, et al., 2005). Partindo do princípio que os pacientes não devem sofrer danos decorrentes da assistência em saúde, o tema da segurança do paciente tem sido norteador do cuidado e recebido destaque em estudos que avaliam a qualidade da assistência em vários países.

Desta forma, o Ministério da Saúde define eventos adversos (EA) como incidentes resultantes da assistência e não relacionados à evolução da doença de base do paciente. Desta forma, ocasionando lesões nos pacientes afetados e aumentando seu tempo de atendimento e internação podendo ocasionar o óbito, ou seja, aumentam a probabilidade de um prognóstico desfavorável na recuperação do indivíduo (CRESPILHO DF, et al., 2015).

Após os Estados Unidos da América divulgarem dados alarmantes de saúde no fim da década de 90, as discussões acerca do tema segurança do paciente tornaram-se frequentes na literatura. O relatório "To Err is Human" revelou um total 44.000 a 98.000 mortes por ano provenientes de erros durante a assistência dos serviços de saúde no país (SOUZA FT, et al., 2014).

Todos os artigos referem que posterior a publicação desses dados, que apontaram as condições de vulnerabilidade a eventos adversos às quais os pacientes estavam expostos durante o processo de cuidado no atendimento pré-hospitalar, as Organizações Nacionais e Internacionais tem investido no estímulo de práticas que promovam maior segurança ao paciente e reduzam os riscos de danos para os mínimos possíveis (SOUZA FT,et al., 2014; CRESPILHO DF, et al., 2015; MACEDO TR, et al., 2016; DANTAS RAN, et al., 2015).

Atualmente, o número estimado de pacientes que morrem anualmente devido a ocorrência de eventos adversos evitáveis, estima-se que esteja em torno de 400 mil e ainda 2 a 4 milhões vivem com sequelas em função da ocorrência de erros durante a assistência e ainda $4,0 \%$ a $16 \%$ do pacientes hospitalizados são acometidos de injúrias relacionadas a eventos adversos em decorrência da assistência prestada. (MACEDO TR, et al., 2016, BRASIL, 2017). 
Dentre os quais, para Souza FT, et al (2014) e Macedo TR, et al (2016), as crianças possuem, pelo menos, três vezes mais risco de sofrerem que os adultos. No ano de 2004, a Organização Mundial de Saúde (OMS), por meio da criação do programa The world alliance for patient safety, procurou efetivas políticas capazes de modificar a assistência ofertada até então nos serviços de saúde ao redor do mundo, assim como, buscando incentivar medidas que orientassem a segurança do paciente (MACEDO TR, 2016).

Contudo, no Brasil, apenas no ano de 2013, o Ministério da Saúde publicou a Portaria no 529, de 2013 que Instituiu o Programa Nacional de Segurança do Paciente (PNSP) visando apoiar e a promover ações voltadas a prestação de uma assistência segura e posteriormente a implantação da gestão de risco e de Núcleos de Segurança do Paciente nos estabelecimentos de saúde, assim como, a obrigatoriedade da notificação dos eventos adversos e a elaboração do Plano de Segurança do Paciente por meio da regulamentação da RDC ํo 36 da Agência Nacional de Vigilância Sanitária (ANVISA) (BRASIL, 2013; CRESPILHO DF, et al., 2015; BRASIL, 2017).

Embora o componente pré-hospitalar móvel da Política nacional de atenção às Urgências exista desde 2003 por meio da Portaria GM № 2072/03, para Crespilho DF (2015) o atendimento de urgência e emergência extra-hospitalar foi pouco abordado pelas políticas de segurança do paciente. Contudo, Dantas RAN, et al. (2015), considera que este serviço pode ser considerado um observatório do funcionamento do Sistema Único de Saúde (SUS), visto que o mesmo atua como um regulador do Sistema, este estudo está em concordância com Crespilho DF, et al. (2015) que afirma essa potencialidade do APH e a necessidade de avaliação constante do serviço a fim de que os erros geradores de Eventos Adversos, quer sejam frequentes ou não, não sejam subnotificados.

Para que a segurança do paciente se torne uma cultura no APH, faz-se necessário que a cultura de segurança esteja estruturada nas instituições, isto significa estabelecer processos de trabalho em equipe com comunicação horizontal, transmissão de confiança, aprendizado organizado por protocolos, compromisso da equipe com os aspectos da segurança e implantação de conduta não punitiva ao erro (MACEDO TR, et al., 2016).

O método punitivo frente à ocorrência do erro é ainda combatido em outro estudo analisado, pois, para os autores o medo perante a punição pode induzir a não-notificação do evento (SOUZA FT, et al., 2014). Um estudo brasileiro que se propôs a avaliar a implantação da cultura de segurança verificou que apesar de haver Núcleos de Segurança e sistemas de notificação em todas as instituições de saúde que participaram da pesquisa, $45 \%$ dos profissionais não fizeram notificações nos últimos 12 meses, e $35 \%$ notificaram até dois eventos adversos. Para os autores, os principais motivos da omissão de comunicação dos eventos adversos ocorridos são medo e vergonha, corroborando com a literatura analisada (CLARO CM, et al., 2011). As principais causas de EA's, apontadas pelos estudos analisados, estão listadas de acordo com o trabalho e autoria no Quadro 2.

Quadro 2 - Distribuição dos estudos de acordo com as principais causas de Eventos Adversos.

\begin{tabular}{|c|c|c|c|}
\hline $\mathbf{N}$ & Título & Autores & Causas de EA'S \\
\hline 1 & $\begin{array}{l}\text { Percepção da enfermagem sobre os } \\
\text { fatores de risco que envolvem a } \\
\text { segurança do paciente pediátrico. }\end{array}$ & SOUZA FT, et al. & $\begin{array}{l}\text { - } \quad \text { Falta de qualificação profissional. } \\
\text { - } \quad \text { Realização de procedimentos } \\
\text { invasivos. }\end{array}$ \\
\hline 2 & $\begin{array}{l}\text { Segurança do paciente na assistência } \\
\text { pré-hospitalar de emergência: uma } \\
\text { revisão integrativa. }\end{array}$ & CRESPILHO DF, et al. & $\begin{array}{l}\text { - } \quad \text { Fator humano (cansaço, estresse, } \\
\text { etc.). } \\
\text { - } \quad \text { Aspectos estruturais. } \\
\text { - } \quad \text { Condições de trabalho. }\end{array}$ \\
\hline 3 & $\begin{array}{l}\text { Cultura de segurança do paciente na } \\
\text { perspectiva da equipe de enfermagem } \\
\text { de emergências pediátricas. }\end{array}$ & MACEDO TR, et al. & $\begin{array}{l}\text { - Falta de estímulo promovido pela } \\
\text { instituição. }\end{array}$ \\
\hline 4 & $\begin{array}{l}\text { Instrumento para avaliação da } \\
\text { qualidade da assistência pré- } \\
\text { hospitalar móvel de urgência: } \\
\text { validação de conteúdo. }\end{array}$ & DANTAS RAN, et al. & $\begin{array}{l}\text { - } \quad \text { Falta de capacitação contínua. } \\
\text { - } \quad \text { Desmotivação para o atendimento. } \\
\text { - } \quad \text { Indisponibilidade de recursos } \\
\text { materiais adequados. }\end{array}$ \\
\hline
\end{tabular}

Fonte: Silva WM, et al., 2020 
A literatura revisada relaciona o aumento de eventos adversos à falta de qualificação profissional e ausência de educação continuada dos profissionais das urgências (SOUZA FT, et al., 2014; CRESPILHO DF, et al., 2015; MACEDO TR, et al., 2016; DANTAS RAN, et al., 2015).

Em três artigos pesquisados, a falta de capacitação contínua é apontada como principal dificuldade enfrentada, no que concerne a segurança do paciente, pois, esta leva a desmotivação para o atendimento, de forma que compromete a qualidade da assistência (SOUZA FT, et al., 2014; MACEDO TR, et al., 2016; DANTAS, et al., 2015).

Macedo TR, et al, (2016) conclui que a assistência segura visando a proteção do paciente é responsabilidade de cada profissional e do estímulo promovido e estabelecido pelas instituições de saúde. Tal estímulo para Crespilho DF, et al. (2015), trata-se de mais que recursos materiais de qualidade, ter à disposição da equipe a possibilidade de treinamento em serviço como uma medida para a superação de dificuldades para a promoção de um atendimento eficiente e de qualidade.

O autor relata ainda que isto se deve ao fato de que o APH é realizado por meio da execução de protocolos e diretrizes. Tal dado está de acordo com os achados de Hagiwara MA, et al. (2013), onde afirma que o trabalho em cumprimento das diretrizes clínicas objetiva melhorar a qualidade da assistência no préhospitalar.

Neste contexto, a ferramenta da simulação utilizada em treinamentos, visa capacitar a equipe na conduta clínica e técnica durante os atendimentos em ambiente extra hospitalar. Embora, o fator humano possa estar presente nos eventos adversos, aspectos estruturais, condições de trabalho e atividades desenvolvidas podem estar relacionadas ao surgimento de erros.

As condições de trabalho também são fatores importantes para a ocorrência de erros no processo do cuidado em APH capazes de gerar dano mensurável no paciente, este dado corrobora o achado de Ebben RHA, et al. (2014) em que ao avaliar 103 serviços de APH móvel de urgência observou que as características físicas, tais como, a conservação da parte mecânica da ambulância e a disponibilidade dos recursos materiais adequados, promovem maior segurança ao paciente durante processo de atendimento e estabilização.

Esta revisão permitiu perceber que os fatores envolvidos nas ocorrências de EA's são de diversas naturezas, fatores estes, que comprometem a assistência a ser prestada ao paciente, podendo levar a sequelas transitórias ou permanentes, o que resulta em custos sociais, além dos custos assistenciais.

Desta forma, este estudo demonstra a necessidade de avanços e melhorias concernentes a institucionalização da cultura de segurança no serviço de $\mathrm{APH}$, por meio da redução de gradientes hierárquicos; fortalecimento dos vínculos de equipe e trabalho comunicativo com horizontalidade entre os membros da equipe e gestores, suficiência de recursos materiais e humanos; fortalecimento do debate acerca do tema segurança do paciente em ambientes de treinamento; substituição da abordagem visando punição pela abordagem sistêmica, como recomenda Macedo TR, et al. (2016); fortalecimento dos sistemas de notificações para que os eventos adversos ocorridos na instituição tornem-se mensuráveis afim de que por meio desses dados estratégias sejam traçadas para que, tais erros, possam ser evitados.

\section{CONSIDERAÇÕES FINAIS}

A contínua capacitação dos profissionais, através de treinamentos em serviços de educação em saúde, apresenta-se como uma estratégia para promover a segurança do paciente nos serviços de urgência e emergência. Nota-se que a padronização das rotinas protocoladas pode se caracterizar como um norteador para promover o alinhamento entre rotinas e prática profissional, de forma a evitar os eventos adversos. Diante do exposto, existe a necessidade de contínua atualização e capacitação dos profissionais, abrindo espaços para a participação e reflexão na busca de alternativas que visam a melhoria da prática. Conclui-se ainda que, existem poucas publicações acerca da temática e reafirma-se a necessidade de realização de estudos que visem o atendimento seguro de pacientes em serviços de urgência e emergência pré-hospitalar. 


\section{REFERÊNCIAS}

1. BRASIL. Ministério da Saúde; Conselho Nacional de Saúde. Resolução n. 466, de 12 de dezembro de 2012. Dispõe sobre diretrizes e normas regulamentadoras de pesquisas envolvendo seres humanos. Brasília; 2012.

2. BRASIL. Ministério da Saúde. Agência Nacional de Vigilância Sanitária. Resolução - RDC no 36 , de 25 de julho de 2013. Institui ações para a segurança do paciente e dá outras providências, Brasília, DF, 2013.

3. BRASIL. Ministério da Saúde. Agência Nacional de Vigilância Sanitária. Resolução - RDC no 36 , de 25 de julho de 2013. Institui ações para a segurança do paciente e dá outras providências, Brasília, DF, 2013.

4. CASTRO GLT, et al. Proposta de passos para a segurança do paciente no atendimento pré-hospitalar móvel. Texto Contexto enfermagem, 2018; 27(3):e3810016.

5. CLARO CM, et al. Adverse events at the Intensive Care Unit: nurses' perception about the culture of no-punishment. Revista Escola de Enfermagem USP, 2011;45(1):162-7.

6. CRESPILHO DF, et al. Segurança do paciente na assistência pré-hospitalar de emergência: uma revisão integrativa. Revista UNINGÁ, 2015; 24(1)45-49.

7. CUNHA VP, et al. Atendimento a pacientes em situação de urgência: do serviço pré-hospitalar móvel ao serviço hospitalar de emergência. Revista Enfermeria Actual, 2019; (37)1-15.

8. EBBEN RHA, et al. An exploration of factors influencing ambulance and emergency nurses' protocol adherence in the Netherlands. Journal of Emergency Nursing, 2014;40(2):124-30.

9. HAGIWARA MA, et al. Decision support system in pre hospital care: arandomized controlled simulation study. American Journal of Emergency Medice, 2013;31(1):145-53

10. INSTITUTE OF MEDICE. To err is human: building a safer health system. Committee on Quality of Health Care in America Washington: National Academy; 2000.

11. MACEDO TR, et al. Cultura de segurança do paciente na perspectiva da equipe de enfermagem de emergências pediátricas. Revista Escola de Enfermagem USP, 2016: 757-763.

12. MENDES W, et al., Revisão dos estudos de avaliação da ocorrência de eventos adversos em hospitais. Revista Brasileira de Epidemiologia, 2005: 8(4): 393-406.

13. OLIVEIRA SN, et al. Emergency Care Units (Upa) 24h: The Nurses' Perception. Texto \& contexto enfermagem, 2015 ;24(1):238-44.

14. PORTO S, et al. A magnitude financeira dos eventos adversos em hospitais no Brasil. Revista Portuguesa de Saúde pública, 2010;(10):74-80

15. REIS CT, et al. Adaptação transcultural da versão brasileira do Hospital Survey On Patient Safety Culture: etapa inicial. Cad Saúde Publica, 2012; 28.

16. RIBEIRO AC e SILVA YB. Pre-hospital nursing in basic life support: ethical and legal postulates of the profession. Cogitare enfermagem, 2016; 21(1):01-8.

17. SANTOS NCM. Urgência e emergência para a enfermagem: do atendimento pré-hospitalar (APH) à sala de emergência. 6ª edição revista e atualizada - São Paulo: látria, 2010.

18. SILVA HC, et al. Trauma in elderly people: access to the health system through pre-hospital care. Revista latinoamericana de enfermagem, 2016; 24: e2690.

19. SOUZA FT, et al. Percepção da enfermagem sobre os fatores de risco que envolvem a segurança do paciente pediátrico. Rev Enferm UFSM, 2014;4(1):152-62.

20. WORLD HEALTH ORGANIZATION. Patient safety research: introductory course - Session 1. Patient safety. 2012. Disponível em: <https://www.who.int/patientsafety/research/online_course/en/ > Acesso em 08 jul. 2020. 